\title{
Cyclin-dependent kinase 1 disruption inhibits angiogenesis by inducing cell cycle arrest and apoptosis
}

\author{
$\mathrm{XIN} \mathrm{GAO}^{1 *}$, YUAN ZHANG $^{1 *}$, RUI ZHANG ${ }^{1 *}$, ZICHAN ZHAO $^{1}$, HAORUI ZHANG $^{2}$, \\ JINHUI WU ${ }^{1}$, WEI SHEN ${ }^{1}$ and MING ZHONG ${ }^{1}$ \\ ${ }^{1}$ Department of Ophthalmology, Shanghai Changhai Hospital; ${ }^{2}$ Company 6 of Basic Medical School, \\ Second Military Medical University, Shanghai 200433, P.R. China
}

Received January 7, 2019; Accepted July 16, 2019

DOI: $10.3892 /$ etm.2019.7883

\begin{abstract}
Angiogenesis is a complex process, which involves the sprouting of new blood vessels from pre-existing vasculature. Pathological retinal angiogenesis can lead to vision loss and even blindness. Cyclin-dependent kinase 1 (CDK1) is involved in regulation of the cell cycle and is reported to contribute to tumor progression. However, the role of CDK1 in retinal angiogenesis is largely unknown. The purpose of the present study was to investigate the role of CDK1 in retinal angiogenesis. Western blotting, reverse transcription-quantitative PCR (RT-qPCR) analysis, immunofluorescence and immunohistochemistry were used to evaluate the expression of CDK1 in pathological angiogenesis using an oxygen-induced retinopathy (OIR) mouse model. Small interfering (si)RNA sequences against CDK1 were synthesized and incubated with retinal cells. The efficiency of knockdown was confirmed by western blot and RT-qPCR assays. The effect of CDK1 siRNAs on angiogenesis in vitro was investigated using EdU cell proliferation, cell migration and tube formation assays. Subsequently, flow cytometry was used to assess the effects of CDK1 siRNAs on cell cycle distribution and on the induction of apoptosis. The expression levels of cell cycle- and apoptosis-related genes were detected using western blotting. CDK1 was overexpressed in pathological retinal angiogenesis. CDK1 siRNAs inhibited human umbilical vein endothelial cell proliferation, migration and tube formation. The possible mechanisms involved the induction of cell cycle arrest at the $\mathrm{G} 2 / \mathrm{M}$ phase and the induction of apoptosis via an increase in the expression levels of p21 and p53. In conclusion, the data
\end{abstract}

Correspondence to: Dr Ming Zhong, Department of Ophthalmology, Shanghai Changhai Hospital, Second Military Medical University, 168 Changhai Road, Shanghai 200433, P.R. China

E-mail: chyanke@126.com

${ }^{*}$ Contributed equally

Key words: angiogenesis, oxygen-induced retinopathy, cyclin-dependent kinase 1 , cell cycle, apoptosis indicated that CDK1 was overexpressed in the OIR model and that silencing of CDK1 inhibited angiogenesis in vitro. CDK1 may be a novel therapeutic target for pathological retinal angiogenesis.

\section{Introduction}

Angiogenesis is the formation of new blood vessels from pre-existing vasculature (1). It is a complex process that involves degradation of the extracellular matrix, the proliferation and migration of endothelial cells and the sprouting of vessels (2). Physiological angiogenesis is of considerable significance in growth, development, wound healing and the normal function of several organs. However, the pathological form of angiogenesis can lead to several diseases, including tumor formation, retinopathy and rheumatoid arthritis $(1,2)$. Angiogenic retinopathy, including diabetic retinopathy, retinopathy of prematurity and retinal vein occlusion, can eventually lead to blindness (3). Although certain therapeutic drugs have been shown to disrupt retinal pathological angiogenesis, several disadvantages have been reported with this type of treatment, including tumor drug resistance and adverse effects (4). Therefore, novel therapeutic targets are required for the treatment of retinal pathological angiogenesis and for investigating the potential molecular mechanisms associated with this process.

Cyclin-dependent kinases (CDKs) are a family of multifunctional enzymes that interact with cyclins to regulate the cell cycle (5). Several checkpoints are present in the cell cycle that ensure accurate cell division. Cyclins bind to CDKs and cause their activation in order to regulate checkpoint transition (6). CDK1 serves an important role in the control of the cell cycle by regulating the centrosome cycle, promoting G2-M transition and modulating G1 phase progression (7). It has also been shown that CDK1 is involved in the regulation of apoptosis $(8,9)$. The cell cycle and apoptosis are well known biological processes that are involved in the development of several pathologies, including tumors and angiogenesis (10). CDK1 is overexpressed in several tumors and contributes to their development (11-14). However, the expression levels and the role of CDK1 in angiogenesis have not been investigated in detail.

The present study investigated the expression levels of CDK1 in pathological angiogenesis using an oxygen-induced 
retinopathy (OIR) mouse model. Furthermore, the effects of CDK1 knockdown on angiogenesis were examined using small interfering (si)RNAs in vitro, and the underlying molecular mechanism was investigated. The present study highlights the role of CDK1 in retinal angiogenesis and may provide novel insight for the development of treatments against pathological neovascularization.

\section{Materials and methods}

Reagents. The antibodies targeting GAPDH (cat. no. 104941-AP), CD31 (cat. no. 11265-1-AP), cyclin A1 (cat. no. 13295-1-AP), cyclin B1 (cat. no. 55004-1-AP), cyclin D1 (cat. no. 60186-1-1 g), cyclin E1 (cat. no. 11554-1-AP), CDK1 (cat. no. 19532-1-AP), CDK2 (cat. no. 10122-1-AP), poly (ADP-ribose) polymerase (PARP)1 (cat. no. 13371-1-AP), p21 (cat. no. 10355-1-AP) and p53 (cat. no. 10442-1-AP) were purchased from Proteintech Group, Inc. DMEM, fetal bovine serum (FBS), DAPI, TRIzol ${ }^{\circledR}$, Super Signal West Pico chemiluminescent substrate, and HRP-(cat. no. A-10677), FITC-(cat. no. A-10683) and Alexa Fluor555-conjugated secondary antibodies (cat. no. A-27017) were obtained from Thermo FisherScientific,Inc.Propidiumiodide(PI) was obtained from Beyotime Institute of Biotechnology. The PrimeScript RT kit and SYBR Premix were purchased from Takara Biotechnology Co., Ltd. (Dalian, China). The Annexin V-FITC apoptosis kit was purchased from eBioscience (San Diego, CA, USA). Griffonia simplicifolia isolectin B4 was purchased from Invitrogen (Thermo Fisher Scientific, Inc.). Matrigel Matrix was purchased from BD Transduction Laboratories (Shanghai, China). The EdU staining kit was obtained from Guangzhou RiboBio Co., Ltd. (Guangzhou, China). Bovine serum albumin was obtained from Weiao Bio (Shanghai, China).

OIR in mice. A total of 32 7-day-old C57BL/6 mice (3.5-3.8 g) with nursing mothers (SIPPR-BK Experimental Animal Co., Ltd., Shanghai, China) were housed under alternate dark-light cycles of $12 \mathrm{~h}$ at room temperature with $50 \%$ humidity and free access to food and water. The sex of mice was not considered a factor for the successful establishment of the OIR model. All surgery was performed under sodium pentobarbital anesthesia, and all efforts were made to minimize the suffering of the animals. The OIR model was used to assess retinal neovascularization, as previously described (15). The newborn mice were housed in a hyperoxic ( $75 \%$ oxygen) chamber connected to an oxygen controller from the first postnatal week (P7-12). At P12, the mice were returned to normoxia. Following exposure to normoxic air for 5 days (P12-17), the mice were sacrificed using carbon dioxide inhalation and their eyes were enucleated and fixed in $4 \%$ paraformaldehyde for $1 \mathrm{~h}$ at room temperature. The retinas were cut four times from the edge to the center and then incubated overnight at $4^{\circ} \mathrm{C}$ with isolectin $\mathrm{B} 4$ in $\mathrm{PBS}$ containing $1 \mathrm{mM} \mathrm{CaCl}_{2}$. Images were captured using an IX81 microscope (Olympus Corporation). All animal experiments were performed with the approval of the Institutional Animal Care and Use Committee of The Second Military Medical University (Shanghai, China).

Immunohistochemistry. The eyes of the mice were enucleated and fixed in $4 \%$ paraformaldehyde for $1 \mathrm{~h}$. Subsequently, the eyeballs were post-fixed in perfusion solution overnight, followed by cryoprotection in $30 \%$ sucrose. The frozen sections of retina were cut to a thickness of $12 \mu \mathrm{m}$ and mounted on glass slides. The retinal sections were blocked with $5 \%$ bovine serum albumin (BSA) for $2 \mathrm{~h}$ at room temperature. Subsequently, the samples were incubated with CDK1 and CD31 antibodies and diluted at a ratio of $1: 200$ in $2 \% \mathrm{BSA}$ overnight at $4^{\circ} \mathrm{C}$. The sections were rinsed with PBS three times and incubated with secondary antibodies diluted at a ratio of 1:200 in PBS for $2 \mathrm{~h}$ at room temperature. The sections were subsequently stained with DAPI, washed and mounted with anti-fading medium. Images were obtained using an IX81 microscope (Olympus Corporation).

Cell culture. Human umbilical endothelial cells (HUVECs), obtained from ScienCell Research Laboratories (Carlsbad, CA, USA), were cultured in DMEM supplemented with $10 \%$ FBS. The cells that were authenticated by cell short tandem repeat genotyping were cultured at $37^{\circ} \mathrm{C}$, in a $5 \% \mathrm{CO}_{2}$ cell incubator. Cells were identified via cell short tandem repeats (STRs) (16). When the cells were grown to $\sim 80 \%$ confluence, they were used for the different assays.

Reverse transcription-quantitative PCR (RT-qPCR) analysis. TRIzol reagent was used to extract the total RNA from mouse retinal tissues and from HUVECs. Subsequently, PrimeScript RT reagent was used to reverse transcribe total RNA to cDNA. SYBR Premix Ex Taq was subsequently used to assess the quantity of cDNA. The reactions were performed using a Rotor Gene 3000A (Corbett Research) instrument. The thermocycling conditions for PCR were as follows: $95^{\circ} \mathrm{C}$ for $2 \mathrm{~min}$, followed by 40 cycles at $95^{\circ} \mathrm{C}$ for $10 \mathrm{sec}, 55^{\circ} \mathrm{C}$ for $30 \mathrm{sec}$ and $72^{\circ} \mathrm{C}$ for $30 \mathrm{sec}$ (17). Each RNA sample was evaluated in triplicate. mRNA levels were subsequently calculated using the $2^{-\Delta \Delta \mathrm{Cq}}$ method (18) and normalized to the expression of GAPDH. The primer sequences $\left(5^{\prime} \rightarrow 3^{\prime}\right)$ used were as follows: TGAGGTAGTAAC ACTCTGGTA (forward) and ATGCTAGGCTTCCTGGTT (reverse) for CDK1 (Homo), TGGGCTACACTGAGCACCAG (forward) and AAGTGGTCGTTGAGGGCAAT (reverse) for GAPDH (Homo), ACTCCAGGCTGTATCTCAT (forward) and CACTCGTATCGGTATTCCAA (reverse) for CDK1 (Mus), CAATGAATAGGGCTACAGCA (forward) and AGG GAGATGCTCAGTGTTGG (reverse) for GAPDH (Mus).

RNA interference. The siRNAs were designed and synthesized by a commercial company (Shanghai GenePharma Co., Ltd., Shanghai, China). The target sequences against CDK1-1 were as follows: Sense, 5'-CCUAUGGAGUUGUGUAUAATT-3' and antisense, 5'-UUAUACACAACUCCAUAGGTT-3'. The target sequences for CDK1-2 were as follows: Sense, 5'-GGA UGUGCUUAUGCAGGAUTT-3' and antisense, 5'-AUC CUGCAUAAGCACAUCCTT-3'. The sequences of scramble siRNA were as follows: Sense, 5'-UUCUCCGAACGUGUC ACGUdTdT-3' and antisense, 5'-ACGUGACACGUUCGG AGAAdTdT. Lipofectamine ${ }^{\circledR} 2000$ was used to transfect siRNAs according to the manufacturer's instructions. Cells were seeded in 24-well plates at a density of $5 \times 10^{3}$ and transfected with $100 \mathrm{ng} / \mu \mathrm{l}$ siRNA. Samples were then incubated for $72 \mathrm{~h}$ at $37^{\circ} \mathrm{C}$ with $5 \% \mathrm{CO}_{2}$. After $72 \mathrm{~h}$, the cells were used for subsequent experimentation. 

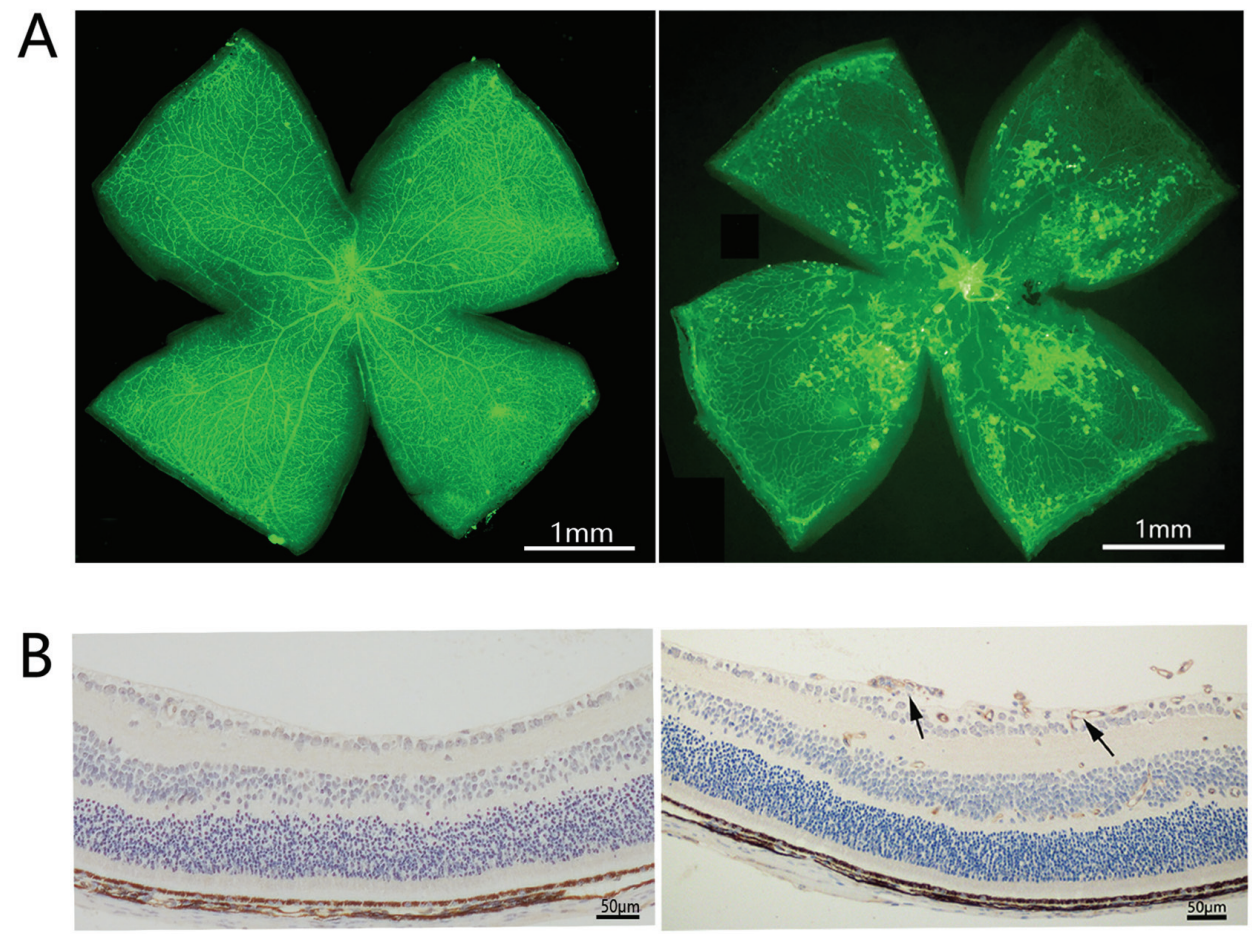

Figure 1. Induction of retinal neovascularization in OIR mice. (A) Representative images of isolectin-B4 staining of the retina in the OIR and normal groups (B) Representative images of immunohistochemical analysis of CD31 in the retina of P17 OIR eyes compared with the control P17 eyes. Black arrows indicate CD31-postive retinal neovascularization. OIR, oxygen-induced retinopathy.

Cell proliferation assay. Cell proliferation was assessed using 5-ethynyl-2'-deoxyuridine (EdU). Following transfection of the cells with CDK1 siRNAs, they were incubated for $72 \mathrm{~h}$ at $37^{\circ} \mathrm{C}$ and their medium was removed. EdU $(100 \mu \mathrm{M})$ was added to the cells and they were incubated for $2 \mathrm{~h}$ at $37^{\circ} \mathrm{C}$. Staining was achieved using a previously described method (17). A total of 4,000 cells, in a total volume of $100 \mu 1$ of Endothelial Cell medium in each well, were incubated overnight at $37^{\circ} \mathrm{C}$ with $5 \% \mathrm{CO}_{2}$. The grown medium was then replaced with medium containing CDK1 siRNAs and incubated for $72 \mathrm{~h}$ at $37^{\circ} \mathrm{C}$. The medium was then discarded and $100 \mu \mathrm{l}$ fresh medium containing EdU (100 mM) was added. Following incubation for $2 \mathrm{~h}$ at $37^{\circ} \mathrm{C}$, the cells were stained according to the following protocol: EdU medium was discarded and the cells were fixed with $4 \%$ paraformaldehyde for $30 \mathrm{~min}$ at room temperature. The cells were then washed with glycine for $5 \mathrm{~min}$, followed by two washes with $0.2 \%$ Triton X-100 (10 min each time). The cells were then stained with Apollo fluorescent azide for $30 \mathrm{~min}$ at room temperature, followed by three washes in $0.2 \%$ Triton X-100. Furthermore, the cells were stained with Hoechst for $30 \mathrm{~min}$ at room temperature and washed with PBS three times, and $100 \mu 1$ PBS was added for further analysis. Images were captured and visualized using an IX81 microscope (Olympus Corporation).

Cell migration assay. A Transwell chamber containing a polycarbonate filter with an $8-\mu \mathrm{m}$ pore size was used to evaluate cell migratory ability. A total of $2 \times 10^{4}$ cells that were treated with CDK1 siRNAs for $72 \mathrm{~h}$ were seeded in the upper chamber in $0.5 \%$ FBS medium. A total of $700 \mu \mathrm{l}$ of DMEM containing $1 \%$ FBS was added to the 24 -well plate. The cells were allowed to migrate for $12 \mathrm{~h}$, and the chambers were then fixed with $4 \%$ paraformaldehyde for $20 \mathrm{~min}$. Subsequently, a cotton swab was used to remove the cells from the upper surface. The chambers were subsequently stained with $0.1 \%$ crystal violet and the migrated cells were counted and analyzed using an IX81 microscope (Olympus Corporation).

Tube formation assay. The 96-well plate was coated with Matrigel and incubated at $37^{\circ} \mathrm{C}$ for $30 \mathrm{~min}$. The cells were treated with CDK1 siRNAs for $72 \mathrm{~h}$ and resuspended in fresh medium. A total of $1.5 \times 10^{4}$ cells were mixed with $100 \mu \mathrm{l}$ of DMEM and added to the Matrigel-coated plates. Following $4 \mathrm{~h}$ of incubation at $37^{\circ} \mathrm{C}$, images of the capillary-like structures were captured and analyzed using an IX81 microscope (Olympus Corporation).

Cell cycle assay. The HUVECs were seeded in a 6-well plate and starved for $16 \mathrm{~h}$. Subsequently, the cells were treated with CDK1 siRNAs for an additional $72 \mathrm{~h}$, harvested and finally fixed in $70 \%$ ethanol at $4^{\circ} \mathrm{C}$ overnight. The ethanol was removed, and the fixed cells were washed twice with PBS. Propidium iodide was used to stain the cells for $30 \mathrm{~min}$ at room temperature. The stained cells were assessed using Cell Lab Quanta SC (Beckman Coulter, Inc.). The data were finally analyzed using Modfit software (MFLT5.0; Verity Software House, Inc.).

Cell apoptosis assay. The HUVECs were seeded in a 6-well plate and starved for $16 \mathrm{~h}$. Subsequently, the cells were treated with CDK1 siRNAs for an additional $72 \mathrm{~h}$ and were then harvested and stained with an Annexin V-FITC apoptosis kit, according to the manufacturer's protocol. The stained cells were assessed using Cell Lab Quanta SC (Beckman Coulter, 


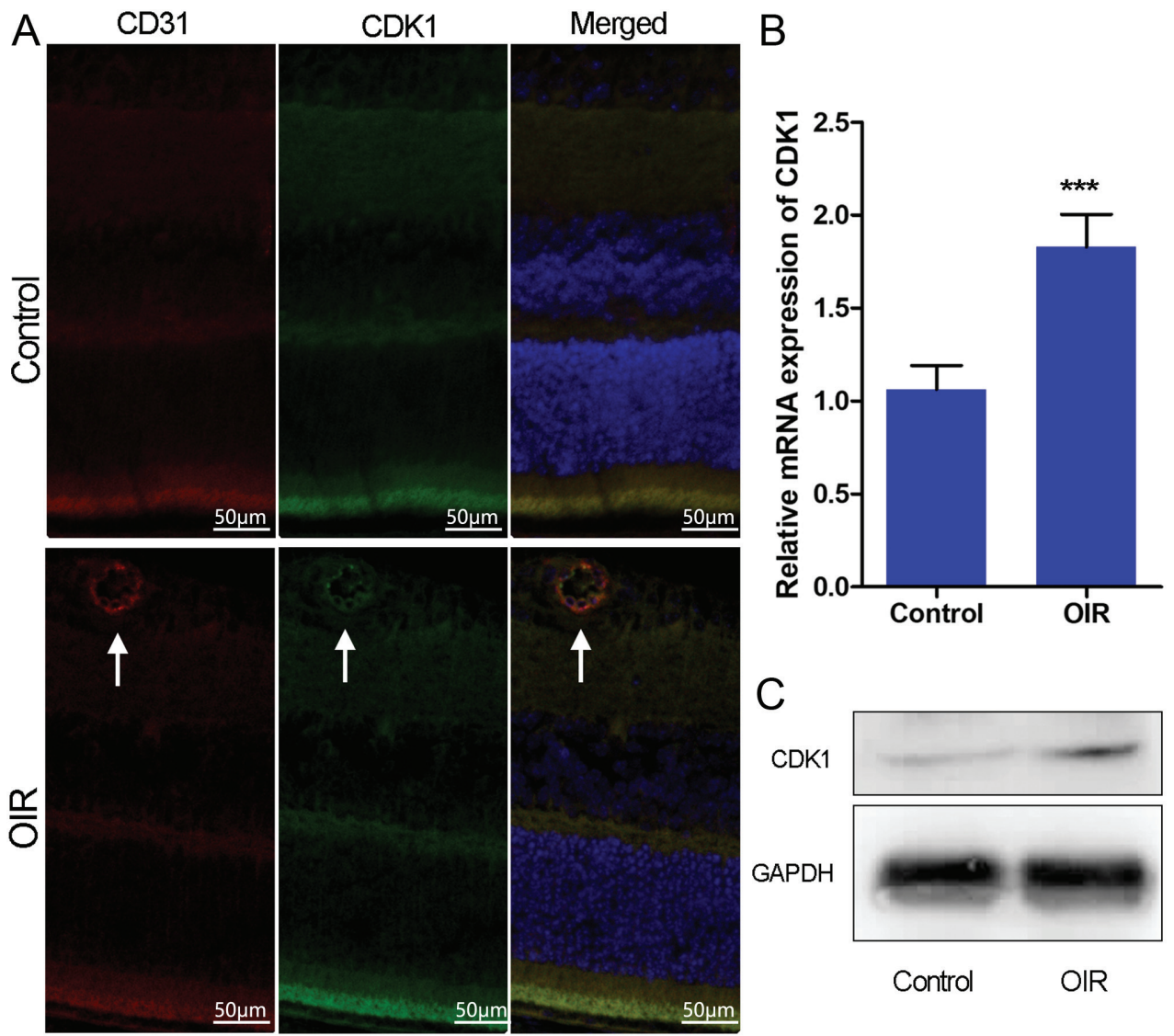

Figure 2. CDK1 is overexpressed in the retina of OIR mice. (A) Representative immunofluorescence analysis of CDK1 in the retina of P17 OIR eyes compared with that noted in control P17 eyes. White arrows indicate CD31 and CDK1 colocalizing with neovascularization in the retinas of OIR mice. (B) mRNA levels of CDK1 from OIR and control retinas. (C) Western blot analysis of CDK1 in OIR and control retinas. GAPDH was used as a loading control. ${ }^{* * *} \mathrm{P}<0.001$. Each value represents the mean \pm SD of three independent experiments. OIR, oxygen-induced retinopathy; CDK1, cyclin-dependent kinase 1 .

Inc.). The data were finally analyzed using FlowJo software (version 7.6.1; FlowJo LLC).

Western blot analysis. Lysis buffer containing protease inhibitor cocktail was used to extract total protein from mouse retinas and HUVECs. The protein concentration of each sample was determined using a bicinchoninic acid protein assay kit (Beyotime Institute of Biotechnology). Approximately $10 \mu \mathrm{g}$ of protein was separated in 10\% SDS/PAGE gels and transferred onto polyvinylidene fluoride membranes. Nonfat milk was used at $5 \%$ to block the non-specific sites. Subsequently, the samples were incubated with GAPDH, CD31, cyclin A1, cyclin B1, cyclin D1, cyclin E1, CDK1, CDK2, PARP1, p21 and p53 primary antibodies diluted at a ratio of 1:1,000 in Universal antibody diluent at $4^{\circ} \mathrm{C}$ overnight, following which the membranes were washed three times with TBS-T and incubated with HRP-conjugated secondary antibodies for $2 \mathrm{~h}$ at room temperature. GAPDH was used as a loading control and the protein signals were finally detected using Super Signal West Pico chemiluminescent substrate and semi-quantified using the GeneGnome HR Image Capture system (Syngene Europe).

Statistical analysis. The data were analyzed and presented using Prism software (GraphPad 5.0; GraphPad Software, Inc.). All data are presented as the mean $\pm \mathrm{SD}$ and the differences between the groups were evaluated using the unpaired t-test or one-way ANOVA. $\mathrm{P}<0.05$ was considered to indicate a statistically significant difference.

\section{Results}

CDK1 is expressed at a high level in pathological retinas. The OIR mouse model is a well-recognized model for the evaluation of retinal angiogenesis. Newborn mice were exposed to hyperoxia between $\mathrm{P} 7$ and P12. On P12, the mice were grown under normoxic conditions. On P17, retinal neovascularization was determined in OIR mice by isolectin-B4 staining and immunohistochemical staining of CD31. In order to investigate the role of CDK1 in retinal angiogenesis, the expression of CDK1 was initially examined in OIR mouse retinas using immunofluorescence staining, RT-qPCR analysis and western blotting. OIR mice exhibited high retinal neovascularization and the immunohistochemical data indicated that $\mathrm{CD} 31$ was overexpressed in the retinas of OIR mice (Fig. 1A and B). Immunofluorescence microscopy demonstrated that the overexpression of CDK1 colocalized with neovascularization in the retinas of OIR mice (Fig. 2A). RT-qPCR and western blot assays demonstrated that the expression levels of CDK1 were significantly higher in the OIR mouse retinas compared with those in the control group (Fig. 2B and C). The data indicated that CDK1 was overexpressed in pathological retinas and, notably, in pathological retinal angiogenesis. 
A

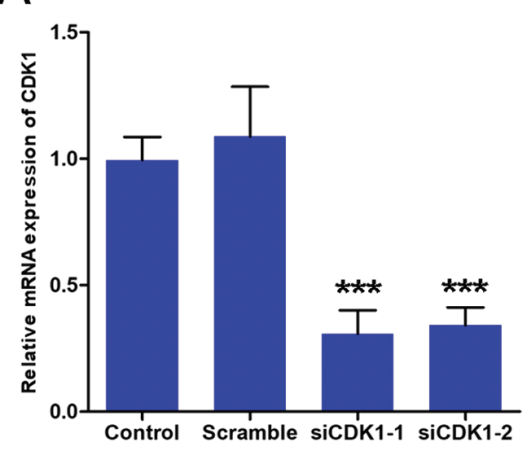

$\mathrm{B}$
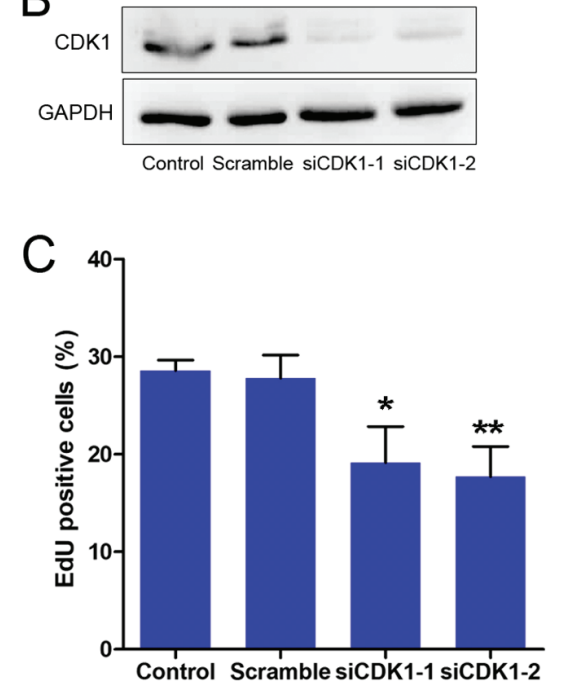

D
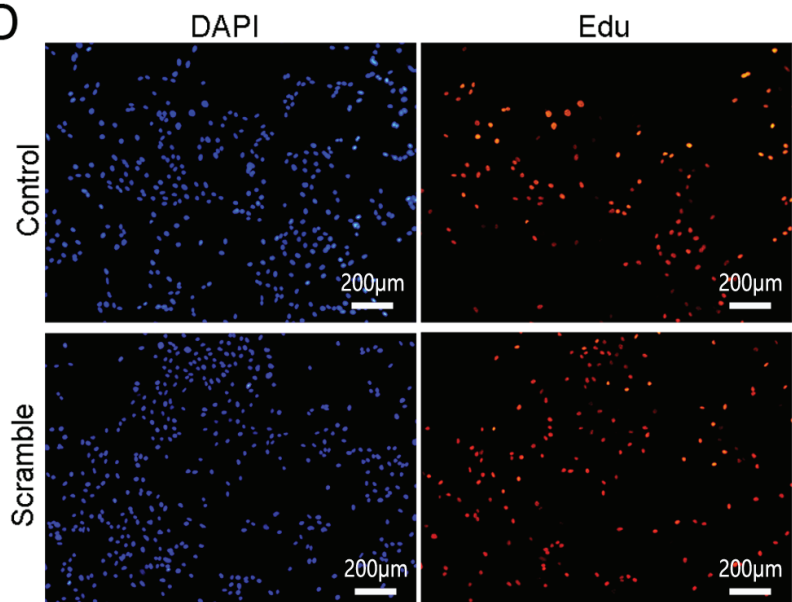

Merged
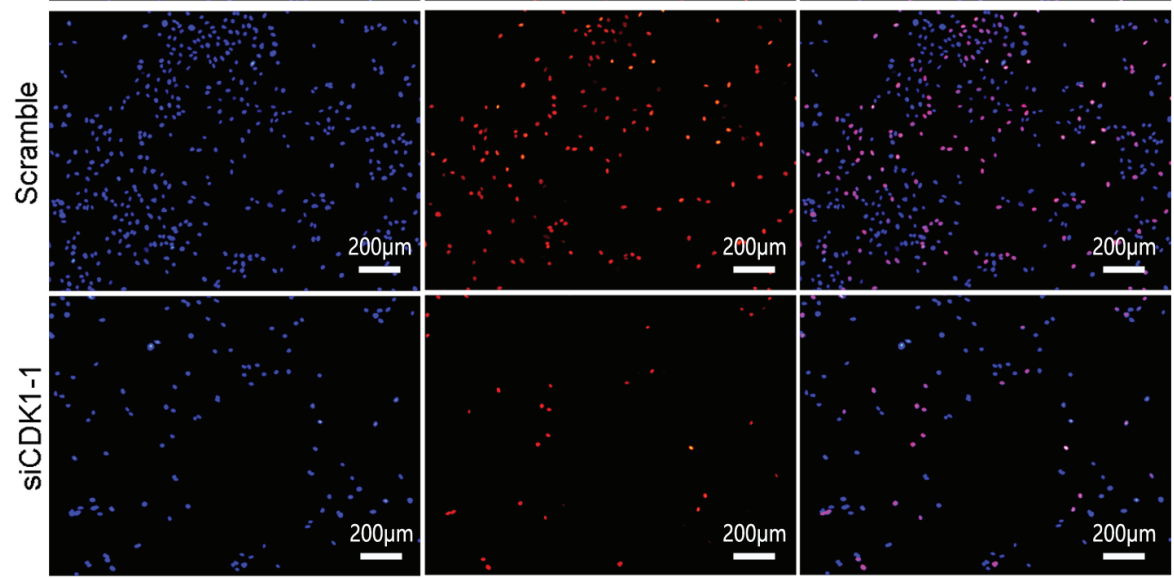
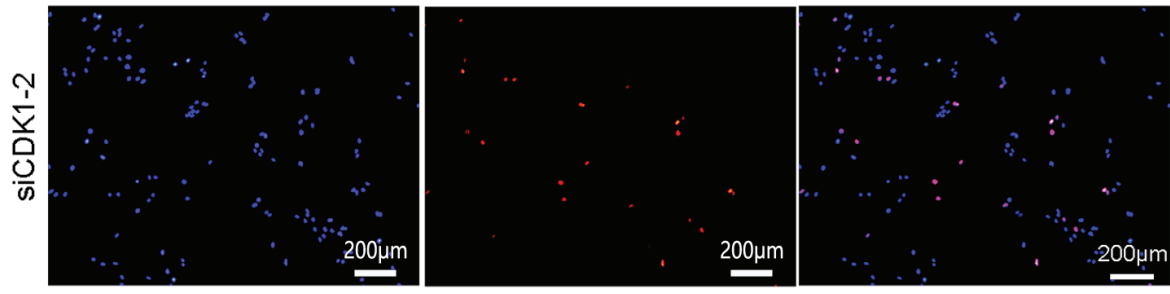

Figure 3. CDK1 siRNAs inhibit endothelial cell proliferation. (A) mRNA levels of CDK1 in the control, scramble and siCDK1 groups. (B) Western blot analysis of CDK1 in the control, scramble and siCDK1 groups. GAPDH was used as a loading control. (C) Quantitative analysis of EdU-positive cells, which represent proliferating cells. (D) Representative images of the cell proliferation assay using EdU. ${ }^{*} \mathrm{P}<0.05,{ }^{* * *} \mathrm{P}<0.01$ and ${ }^{* * * *} \mathrm{P}<0.001$ vs. the control group. Each value represents the mean \pm SD of three independent experiments. CDK1, cyclin-dependent kinase 1; siRNA, small interfering RNA.
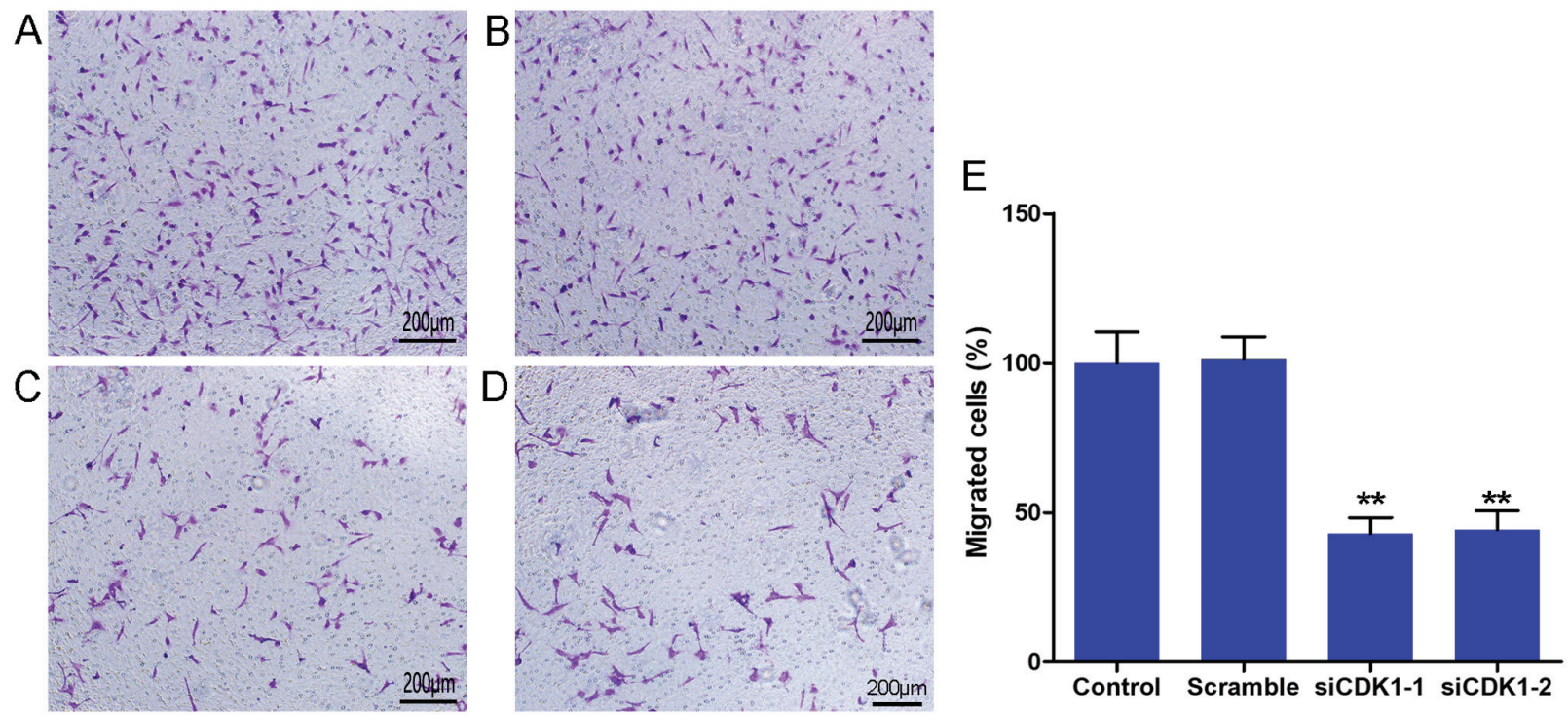

Figure 4. CDK1 siRNAs inhibit endothelial cell migration. Representative images of migrated cells in the (A) control, (B) scramble, (C) siCDK1-1 and (D) siCDK1-2 groups using the Transwell chamber assay. (E) Quantitative analysis of migrated cells. ${ }^{* *} \mathrm{P}<0.01$ vs. the control group. Each value represents the mean \pm SD of three independent experiments. CDK1, cyclin-dependent kinase 1; siRNA, small interfering RNA.

Silencing of CDK1 inhibits HUVEC growth. To further investigate the role of CDK1 in angiogenesis, CDK1 siRNAs were synthesized and their knockdown efficiency was assessed. The results indicated that the CDK1 siRNAs significantly 

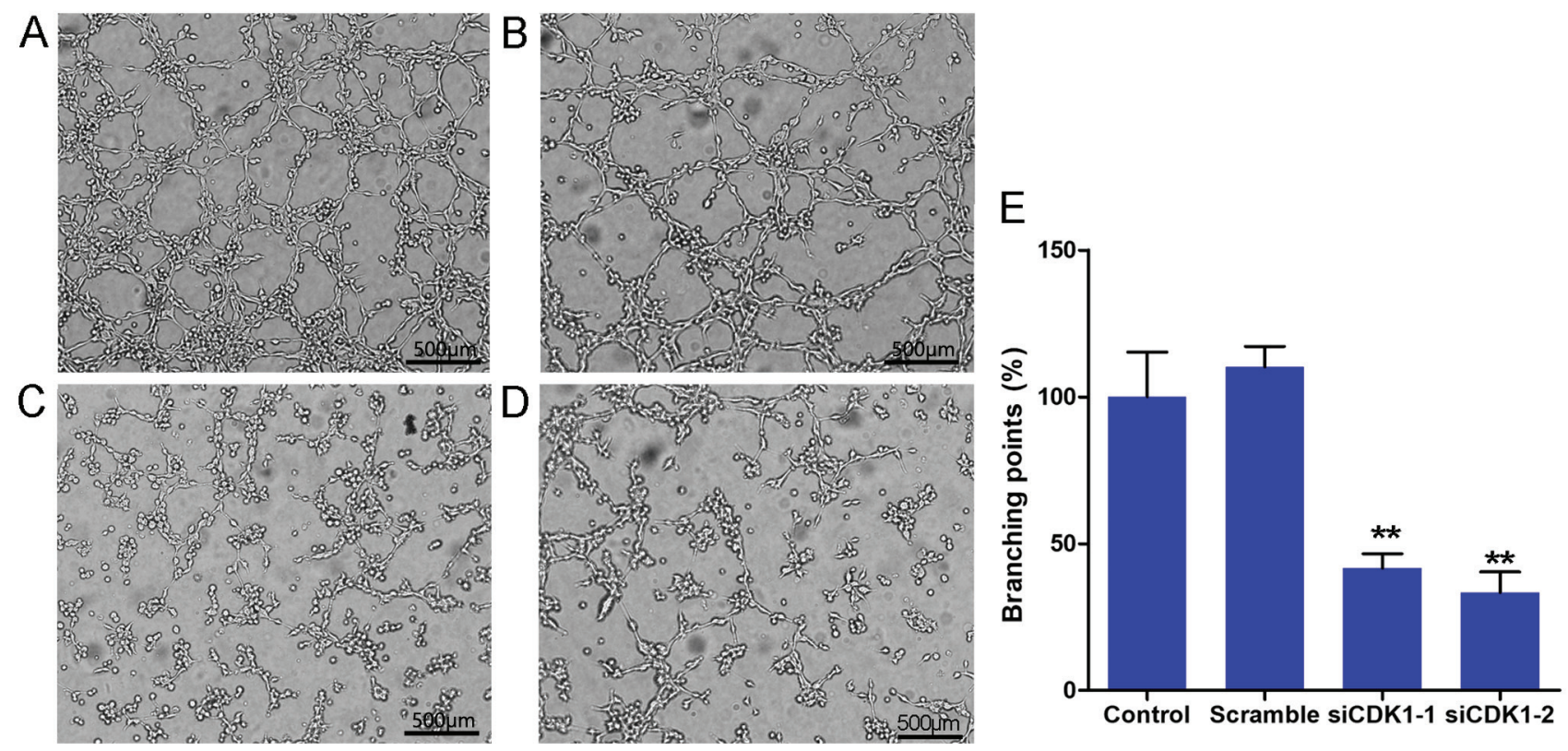

Figure 5. CDK1 siRNAs inhibit endothelial cell tube formation. Representative images of tube formation in the (A) control, (B) scramble, (C) siCDK1-1 and (D) siCDK1-2 groups. (E) Quantitative analysis of tube branching points. ${ }^{* *} \mathrm{P}<0.01$ vs. the control group. Each value represents the mean $\pm \mathrm{SD}$ of three independent experiments. CDK1, cyclin-dependent kinase 1; siRNA, small interfering RNA.

inhibited the expression of CDK1 at the mRNA and protein levels (Fig. 3A and B). The effect of CDK1 siRNAs on cell proliferation was subsequently investigated by EdU. The data indicated that the cells treated with CDK1 siRNAs proliferated more slowly than those of the control and scramble groups (Fig. 3C and D). The data indicated that the transfection was efficient and that the knockdown of CDK1 may exert anti-angiogenic activity, partially through inhibition of the growth of vascular endothelial cells.

Silencing of CDK1 inhibits the migration of HUVECs. The Transwell chamber assay was used to determine cell migration. The results indicated that CDK1 siRNAs inhibited the number of migrated cells significantly compared with that in the control and the scramble groups (Fig. 4A-D). No differences were observed between the control and the scramble groups (Fig. 4E). The number of migrated cells in the siCDK1-1 group was decreased to $57.1 \%$ compared with that of the control group, whereas the number of migrated cells in the siCDK1-2 group was decreased to $55.7 \%$ compared with that of the control group (Fig. 4E). The data suggested that silencing of CDK1 inhibited vascular endothelial cell migration, which is considered a vital process for angiogenesis.

Silencing of CDK1 inhibits the tube formation of HUVECs. The tube formation assay is a well-known method to evaluate the angiogenic activity of endothelial cells in vitro. In the present study, tube formation assays were performed using Matrigel. The results indicated that CDK1 siRNAs significantly inhibited tube formation of HUVECs compared with that noted in the control and scramble groups (Fig. 5A-D). No significant difference was noted with regard to tube formation between the control and scramble groups (Fig. 5E). The percentage of branching points in the siCDK1-1 group decreased to $58.3 \%$ compared with that of the control group, and the percentage of migrated cells in the siCDK1-2 group decreased to $66.7 \%$ compared with that of the control group (Fig. 5E). The data suggested that CDK1 served an important role in angiogenesis, and that knockdown of CDK1 inhibited angiogenesis in vitro.

Silencing of CDK1 inhibits the cell cycle and apoptosis. The effects of CDK1 knockdown on cell cycle progression and the induction of apoptosis were investigated. The knockdown of CDK1 induced cell cycle arrest at the G2/M phase (Fig. 6A). The percentage of the cell population in the G2/M phase increased by $\sim 10 \%$ in both the siCDK1-1 and siCDK1-2 groups compared with that noted in the control group (Fig. 6B). Furthermore, the knockdown of CDK1 induced apoptosis compared with that in the control and scramble groups (Fig. 6C). The percentages of early and late apoptotic cells in the CDK1 knockdown groups increased by $\sim 9 \%$ compared with those of the control group (Fig. 6D). Subsequently, the expression levels of cell cycle- and apoptosis-related proteins were investigated using western blot analysis. The results indicated that silencing of CDK1 inhibited the expression levels of cyclin B, cyclin D and CDK2, and increased the expression levels of cyclin A, cyclin E, PARP1, p21 and p53 (Fig. 7A and B).

\section{Discussion}

Previous studies have revealed that CDK1 was expressed at a high level in several types of tumor tissues and that it served an important role in the incidence and development of tumors $(12,14,19)$. However, the role of CDK1 in angiogenesis has not been fully investigated. The formation of pathological angiogenesis resembles the formation of tumor angiogenesis, and both of these phenomena are characterized by the uncontrolled growth of multiple cells (20). In addition, tumor growth is usually accompanied by angiogenesis, which 
A

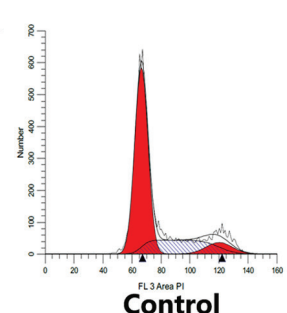

Control

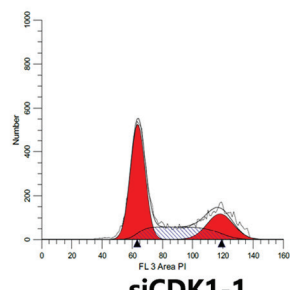

siCDK1-1

C
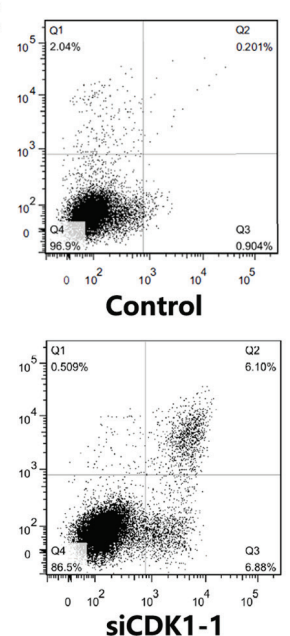

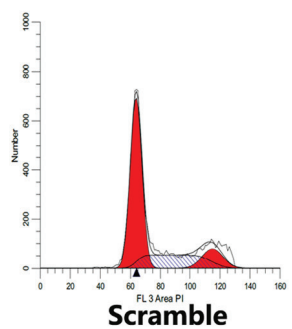

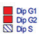
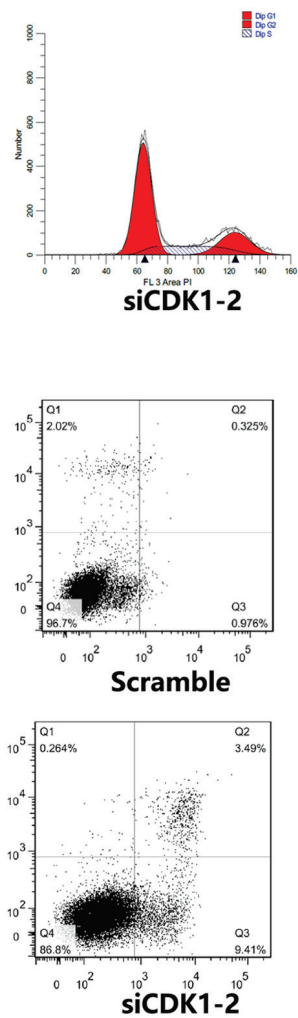

$B$
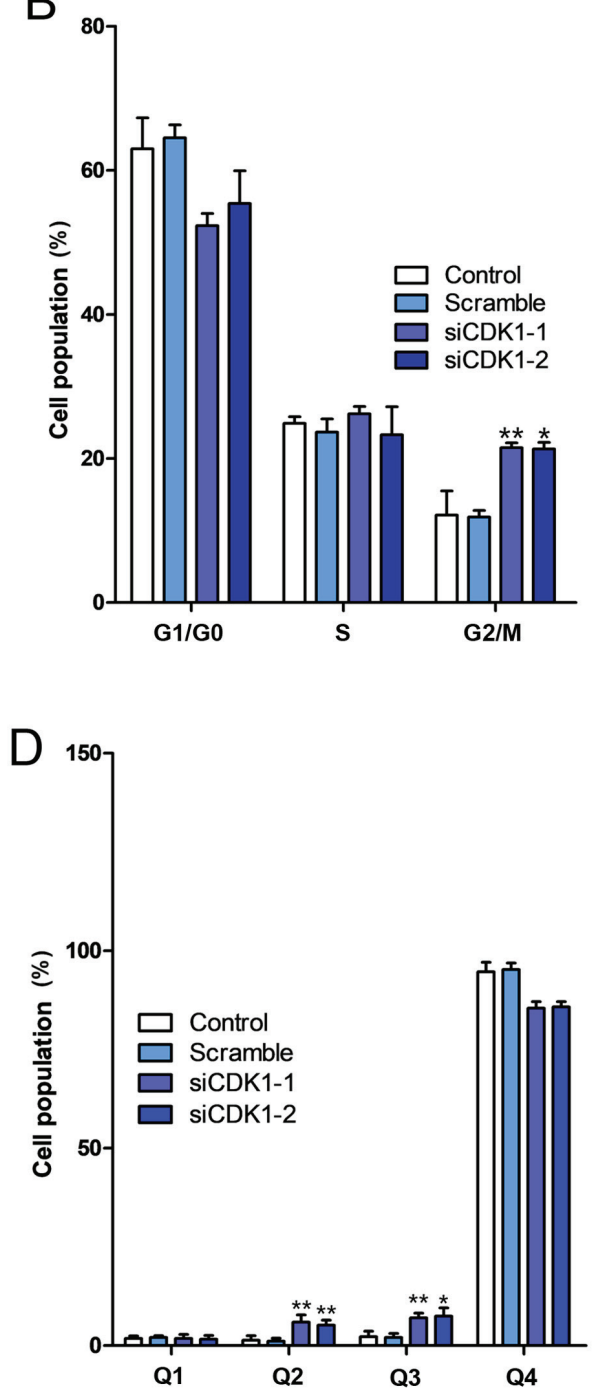

Figure 6. CDK1 siRNAs inhibit angiogenesis by inducing cell cycle arrest. (A) Representative images of cell cycle analysis. (B) Quantitative analysis of the cell cycle population. (C) Representative images of cell apoptotic assay. Q1 represents non-viable cells, Q2 represents late apoptotic cells, Q3 represents early apoptotic cells and Q4 represents viable cells. (D) Quantitative analysis of the result of the cell apoptosis assay. ${ }^{*} \mathrm{P}<0.05$ and ${ }^{* * *} \mathrm{P}<0.01$ vs. the control group. Each value represents the mean \pm SD of three independent experiments. CDK1, cyclin-dependent kinase 1; siRNA, small interfering RNA.

A

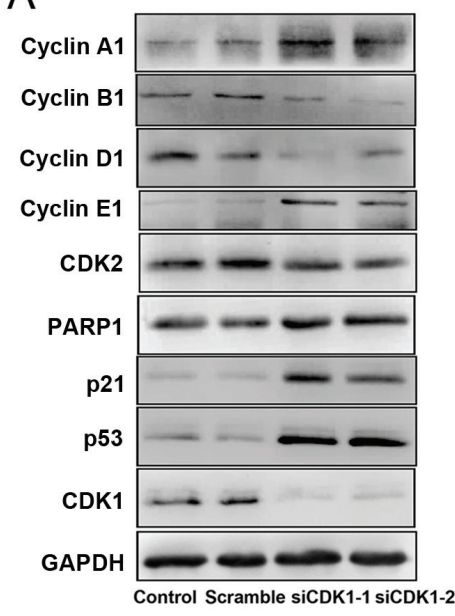

B

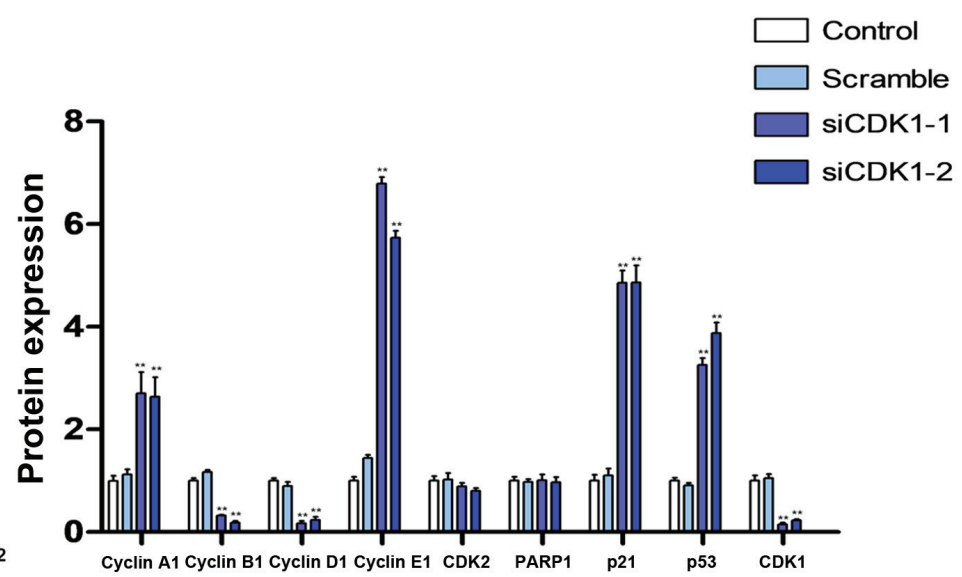

Figure 7. Silencing of CDK1 increases the expression levels of p53 and p21. (A) Western blotting indicating the effects of siCDK1 on the expression levels of cyclin A1, cyclin B1, cyclin D1, cyclin E1, CDK2, PARP1, p21 and p53. (B) Semi-quantification of western blotting results. ${ }^{* *} \mathrm{P}<0.01$ vs. the control group. Each value represents the mean $\pm \mathrm{SD}$ of three independent experiments. CDK1, cyclin-dependent kinase 1; PARP1, poly (ADP-ribose) polymerase 1; siRNA, small interfering RNA. 
provides oxygen and nutrients to the tumor tissue (20). It has been reported that CDK1 can stabilize hypoxia-inducible factor- $1 \alpha(\mathrm{HIF}-1 \alpha)$ in order to promote tumor angiogenesis and that HIF-1 $\alpha$ is an important molecule that initiates the angiogenic cascade (14). These findings suggest that CDK1 may be involved in the formation of pathological angiogenesis.

Pathological retinal angiogenesis is a severe disorder, which can result in retinal detachment, optic neuropathy and glaucoma $(21,22)$. These diseases can cause vision loss and even blindness. The vascular endothelial growth factor (VEGF) antibody has been used clinically as an inhibitor of retinal angiogenesis. However, subsequent studies have demonstrated that VEGF may cause cardiovascular adverse effects and that certain patients can develop drug resistance (23). Therefore, the identification of novel therapeutic targets is required to treat retinopathy in combination with the VEGF antibody and/or independently. In the present study, it was demonstrated for the first time, to the best of our knowledge, that CDK1 was overexpressed in pathological retinal tissues. The overexpression of CDK1 occurred concomitantly with neovascularization, as demonstrated by the colocalization of protein expression. This finding indicated that CDK1 served an important role in the formation of pathological angiogenesis. The cell proliferative, migratory and tube formation assays further suggested that targeting of CDK1 may be an important strategy to improve retinal neovascularization.

Although CDK1 is known to regulate the cell cycle process, it serves a multitude of roles in different tumor microenvironments. The silencing of CDK1 induced HCC 827 cell cycle arrest at the G1 phase, whereas the silencing of CDK1 induced HeLa cell cycle arrest at the G2/M phase $(12,24)$. These two cell lines are of human non-small cell lung cancer and human cervical cancer origin, respectively. The present study indicated that silencing of CDK1 induced endothelial cell cycle arrest at the $\mathrm{G} 2 / \mathrm{M}$ phase. The CDK1-cyclin B1 complex, also known as M-phase-promoting factor is particularly relevant with regard to mitotic progression (25-27). The data obtained in the present study indicated that the expression of cyclin B1 was inhibited in the CDK1 siRNA group. In addition, the expression levels of the cell cycle related genes, namely cyclin A1, cyclin D1, cyclin E1 and CDK2, were significantly altered. These may partially account for the cell cycle arrest induced by CDK1 siRNAs. Apoptosis is an additional biological process that requires the involvement of CDK1 $(28,29)$. The results of the present study indicated that the silencing of CDK1 induced apoptosis and increased the expression of PARP1. These data were consistent with previous findings demonstrating that CDK1 protected mitotic cells against the induction of apoptosis (8). Furthermore, the present study showed that the silencing of CDK1 increased the expression levels of p53 and p21. p53 and p21 are two key genes that regulate the cell cycle and the apoptotic process (30-32). These data indicate that the silencing of CDK1 may activate p21 and p53 in order to induce endothelial cell cycle arrest and apoptosis.

In the present study, the expression of CDK1 in OIR, its role in angiogenesis in vitro and the possible mechanism underlying this process were investigated for the first time. The data indicated that the expression of CDK1 was high in pathological retinal angiogenesis and that CDK1 siRNAs inhibited endothelial cell proliferation, migration and tube formation.
Furthermore, CDK1 siRNAs induced cell cycle arrest and apoptosis. The possible mechanism was associated with inhibition of the expression of cell cycle- and apoptosis-related proteins mediated by increasing the protein expression levels of the p21 and p53. Collectively, the results highlight the role of CDK1 in retinal angiogenesis and suggest its potential application for pathological neovascularization therapy.

\section{Acknowledgements}

Not applicable.

\section{Funding}

The present study was supported by the National Natural Science Foundation of China (grant. no. 81700839), the Shanghai Municipal Planning Commission of Science and Research Fund (grant. no. 20170054), the Research Foundation for Youth of Second Military Medical University (grant. no. 2016QN13) and the Shanghai Pujiang Program (grant. no. 17PJD041).

\section{Availability of data and materials}

All data generated or analyzed during the current study are included in this published article.

\section{Authors' contributions}

$\mathrm{XG}, \mathrm{YZ}$ and RZ conceived and designed the experiments, and wrote the manuscript. HZ, ZZ and JW acquired, analyzed and interpreted the data. WS and MZ conceived the current study and revised the manuscript for intellectual content. All authors read and approved the final manuscript.

\section{Ethics approval and consent to participate}

All animal experiments were performed with the approval of the Institutional Animal Care and Use Committee of The Second Military Medical University (Shanghai, China).

\section{Patient consent for publication}

Not applicable.

\section{Competing interests}

The authors declare that they have no competing interests.

\section{References}

1. Potente $\mathrm{M}$ and Carmeliet $\mathrm{P}$ : The link between angiogenesis and endothelial metabolism. Annu Rev Physiol 79: 43-66, 2017.

2. Usui Y, Westenskow PD, Murinello S, Dorrell MI, Scheppke L, Bucher F, Sakimoto S, Paris LP, Aguilar E and Friedlander M: Angiogenesis and eye disease. Annu Rev Vis Sci 1: 155-184, 2015.

3. Rubio RG and Adamis AP: Ocular angiogenesis: Vascular endothelial growth factor and other factors. Dev Ophthalmol 55: 28-37, 2016.

4. Tah V,Orlans HO,HyerJ,CasswellE, Din N, Sri Shanmuganathan V, Ramskold L and Pasu S: Anti-VEGF therapy and the retina: An update. J Ophthalmol 2015: 627674, 2015. 
5. Malumbres $\mathrm{M}$ and Barbacid M: Cell cycle, CDKs and cancer: $\mathrm{A}$ changing paradigm. Nat Rev Cancer 9: 153-166, 2009.

6. Lim S and Kaldis P: Cdks, cyclins and CKIs: Roles beyond cell cycle regulation. Development 140: 3079-3093, 2013.

7. Asghar U, Witkiewicz AK, Turner NC and Knudsen ES: The history and future of targeting cyclin-dependent kinases in cancer therapy. Nat Rev Drug Discov 14: 130-146, 2015.

8. Allan LA and Clarke PR: Phosphorylation of caspase-9 by CDK1/cyclin B1 protects mitotic cells against apoptosis. Mol Cell 26: 301-310, 2007.

9. Matthess Y, Raab M, Knecht R, Becker S and Strebhardt K Sequential Cdk1 and Plk1 phosphorylation of caspase- 8 triggers apoptotic cell death during mitosis. Mol Oncol 8: 596-608, 2014

10. Pietenpol JA and Stewart ZA: Cell cycle checkpoint signaling: Cell cycle arrest versus apoptosis. Toxicology 181: 475-481, 2002.

11. Zeng Y, Stauffer S, Zhou J, Chen X, Chen Y and Dong J: Cyclin-dependent kinase 1 (CDK1)-mediated mitotic phosphorylation of the transcriptional co-repressor Vgll4 inhibits its tumor-suppressing activity. J Biol Chem 292: 15028-15038, 2017

12. Pu S, Zhao Y, Zhou G, Zhu H, Gong L, Zhang W, Huang G, Wang D and Liu D: Effect of CDK1 shRNA on proliferation, migration, cel cycle and apoptosis in non-small cell lung cancer: Effect of CDK1 shRNA on NSCLC cells. J Cell Physiol 233: 7514, 2017.

13. Manchado E, Guillamot M, de Cárcer G, Eguren M, Trickey M, García-Higuera I, Moreno S, Yamano H, Cañamero $M$ and Malumbres M: Targeting mitotic exit leads to tumor regression in vivo: Modulation by Cdk1, Mastl, and the PP2A/B55 $\alpha, \delta$ phosphatase. Cancer Cell 18: 641-654, 2010.

14. Warfel NA, Dolloff NG, Dicker DT, Malysz J and El-Deiry WS CDK1 stabilizes HIF-1 $\alpha$ via direct phosphorylation of Ser668 to promote tumor growth. Cell Cycle 12: 3689-3701, 2013.

15. Connor KM, Krah NM, Dennison RJ, Aderman CM, Chen J, Guerin KI, Sapieha P, Stahl A, Willett KL and Smith LE: Quantification of oxygen-induced retinopathy in the mouse: A model of vessel loss, vessel regrowth and pathological angiogenesis. Nat Protoc 4: 1565-1573, 2009.

16. Guo T, Song H, Zhao Z, Qi Z and Zhao S: Overexpression of Annexin A2 Receptor inhibits neovascularization via the promotion of Krüppel-Like transcription factor 2. Cell Physiol Biochem 46: 1617-1627, 2018.

17. Song H, Wang W, Zhao P, Qi Z and Zhao S: Cuprous oxide nanoparticles inhibit angiogenesis via down regulation of VEGFR2 expression. Nanoscale 6: 3206-3216, 2014.

18. Livak KJ and Schmittgen TD: Analysis of relative gene expression data using real-time quantitative PCR and the 2(-Delta Delta C(T)) method. Methods 25: 402-408, 2001.

19. Sung WW, Lin YM, Wu PR, Yen HH, Lai HW, Su TC, Huang RH, Wen CK, Chen CY, Chen CJ and Yeh KT: High nuclear/cytoplasmic ratio of Cdk1 expression predicts poor prognosis in colorectal cancer patients. BMC Cancer 14: 951, 2014
20. Ronca R, Benkheil M, Mitola S, Struyf S and Liekens S: Tumor angiogenesis revisited: Regulators and clinical implications. Med Res Rev 37: 1231-1274, 2017.

21. Campochiaro PA: Molecular pathogenesis of retinal and choroidal vascular diseases. Prog Retin Eye Res 49: 67-81, 2015.

22. Puro DG, Kohmoto R, Fujita Y, Gardner TW and PadovaniClaudio DA: Bioelectric impact of pathological angiogenesis on vascular function. Proc Natl Acad Sci USA 113: 9934-9939, 2016.

23. Simó R and Hernandez C: Intravitreous anti-VEGF for diabetic retinopathy: Hopes and fears for a new therapeutic strategy. Diabetologia 51: 1574, 2008.

24. Xiao H, Tian M, Ge J, Wei X, Li Z, Li X, Tao D, Hu J and Gong J: The role of CDK1 siRNA interference in cell cycle and cell apoptosis. Front Med China 3: 384, 2009

25. Rattani A, Vinod PK, Godwin J, Tachibana-Konwalski K, Wolna M, Malumbres M, Novák B and Nasmyth K: Dependency of the spindle assembly checkpoint on Cdk1 renders the anaphase transition irreversible. Curr Biol 24: 630-637, 2014

26. Jang SH, Kim AR, Park NH, Park JW and Han IS: DRG2 regulates $\mathrm{G} 2 / \mathrm{M}$ progression via the cyclin $\mathrm{B} 1-\mathrm{Cdk} 1$ complex. Mol Cells 39: 699-704, 2016.

27. Wang Z, Fan M, Candas D, Zhang TQ, Qin L, Eldridge A Wachsmann-Hogiu S, Ahmed KM, Chromy BA, Nantajit D, et al: Cyclin $\mathrm{B} 1 / \mathrm{Cdk} 1$ coordinates mitochondrial respiration for cell-cycle G2/M progression. Dev Cell 29: 217-232, 2014.

28. Zhang P, Kawakami H, Liu W, Zeng X, Strebhardt K, Tao K, Huang $S$ and Sinicrope FA: Targeting CDK1 and MEK/ERK overcomes apoptotic resistance in BRAF-mutant human colorectal cancer. Mol Cancer Res 16: 378-389, 2018.

29. Zhang R, Shi H, Ren F, Zhang M, Ji P, Wang W and Liu C: The aberrant upstream pathway regulations of CDK1 protein were implicated in the proliferation and apoptosis of ovarian cancer cells. J Ovarian Res 10: 60, 2017.

30. Karimian A, Ahmadi Y and Yousefi B: Multiple functions of $\mathrm{p} 21$ in cell cycle, apoptosis and transcriptional regulation after DNA damage. DNA Repair (Amst) 42: 63-71, 2016.

31. Qi LW, Zhang Z, Zhang CF, Anderson S, Liu Q, Yuan CS and Wang CZ: Anti-colon cancer effects of 6-shogaol through G2/M cell cycle arrest by p53/p21-cdc2/cdc25A crosstalk. Am J Chin Med 43: 743-756, 2015.

32. Chen J: The cell-cycle arrest and apoptotic functions of $\mathrm{p} 53$ in tumor initiation and progression. Cold Spring Harb Perspect Med 6: a026104, 2016.

This work is licensed under a Creative Commons Attribution-NonCommercial-NoDerivatives 4.0 International (CC BY-NC-ND 4.0) License. 